\title{
Studies on the role of microtubules in myofibrillogenesis
}

\author{
LIN ZhongXiang AND How ARD HOLTZEN*
}

Depactment of Cell Biology, Beijing Institute for Cancer Research, Beijing, China.

*Depactment of Anatomy, School of Medicine, University of Pennsylvania, Philadelphia, PA, USA.

\begin{abstract}
Go-localization of microtubnle (MT) and muscle myosin (MHC) myofibril immunofluoreseoneo in developing myotubes of chicken skeletal muscle cultures was observed by using double staining of tubulin and MHC indirect immunofluorescence. 12o-tetradecanoyl-phorbol-13-acetate (TPA) selectively and reversibly blocks myofibrillogenesis and alters the morphology of myotubes into myosacs where MTs are present in radiating pattern. When the arrested myogenic cells recover and start myofibrillogenesis after released from TPA, prior to the emergence of myofibrils, the pro-existing MTs become bipolarly aligned eoineidontly with the tubular restoration of cell shape. Single nascent myofibrils overlapping with MTs extend into the base of growth tips where MTs go farther to the end of the tips. That MT might act as scaffold in guiding the bipolar elongation of the growing myofibrils was suggested. Taxol and eoleomid disturbed MT polymerization and disposition, and interfered with the normal spatial assembly of myofibrils in developing myotubos.
\end{abstract}

Key words: Microtubules, myosin heavy chain (MHC), doubled indirect immunofluorescence, myofibrillogenesis.

\section{INTRODUCTION}

During myofibrillogenesis, definitive myogenic cells selectively synthesize a complex set of contractile proteins that interact precisely to form relatively invariant striated myofibril. Those myofibrillar isoforms are assembled into definitive sarcomeres which are arranged in tandem during the earliest stages of myofibrillogenesis in vivo and in vitro [1]. With maturation, nascent myofibrils increase in size; via addition of interdigitating thickand thin-filaments. The increasing new sarcomeres are added at the ends of the elongating myofibrils as has been observed immunocytochemically and electron microscopically [2].

1 Correspondence: LIN Z Da-Hong-Luo-Ghang St., West District. Beijing 100034, China 


\section{Microtubules and myofibrillogenesis}

An intriguing aspect of myofibrillogenesis is that a set of non-muscle constitutive (contractile and non-contractile) proteins as well as the muscle specific contractile protein isoforms are synthesized in postmitotic myoblasts and myotubes. Attention has been drawn upon to the role of non-muscle constitutive proteins in myofibrillogenesis; no obvious role for desmin IFs in the early assembly of striated myofibrils in skeletal muscle has been documented [3]. However, there is evidence that the earliest stages of myofibrillogenesis involve the non-muscle contractile isoforms that constitute stress fibers and miorofilaments; the stress fiber-like struotures (SFLS) which function as precursors and transitory nucleating sites for nascent myofibrils are transient structures in immature myogenic cells and in growth tips of myotubes [1, 4]. On the other hand, less knowledge has been accumulated concerning the role of MTs in myofibrillogenesis. MTs exist in immature - and mature myogenic cells. Earlier work demonstrated that intact MTs were required for the retention of anisodiametrical morphology of myotubes and probably for their normal elongation [3]. That MTs might play roles in deploying thick filaments during myofibril formation was suggested by EM observations [4]. MT disturbing drugs, taxol and colcemid caused remarkable changes in cell shape and the formation of hybrid myofibrils [4]. MT is responsible for elongation or spreading of myotubular growth tips in vitro[5]. However, the temporal and topographical correlation between MTs and emerging myofibrils during the maturation of myofibrils especially in early stages of myofibril formation has yet to be elucidated.

In this investigation, we examined the correlation between the distribution of MTs and the emerging myofibrils immunofluorescently in early stages of myofibrillogenesis of chick embryonic skeletal muscle cultures. To further explore the possible role of MTs in assembly of myofibrils, we took advantage of TPA treated myotubes. TPA selectively and reversibly blocks myofibril formation and alters the form of myotubes into sac-like shape, the myosacs[2]. the TPA-myosacs recover rapidly and form typically striated myofibrils when they are transferred to normal medium and thus have provided an ideal system for studying the role of different classes of cytoskeletal elements during various stages of myofibrillogenesis[2]. The possible role of MTs has been investigated through taxol and colcemid effect on the recovery of TPA-myosacs[3, 4] (mentioned above). Here, further information from double staining with anti-tubulin and anti-MHC immunofluorescence as well as EM results is added. In both normal and TPA-myosac recovering myogenic cells, distribution of MTs changed coincidently with myotube development, from radiating to longitudinal pattern, suggesting bhat mechanisms controlling the organization of MTs were subjected to the cell-lineage specific differentiation program of myogenic cells. Colocalization of MTs with myofibrils strongly suggested that MTs play as scaffold in supporting myofibril formation and in guiding bipolar elongation of growing myofibrils In developing myotubes, disturbance of MT polymerization and disposition interfered with the normal spatial assembly and elongation of myofibrils.

\section{MATERIALS AND METHODS}

\section{Cell culture}

Primary cultures of chicken breast muscle cells from day-12 embryos were used. 
Previously reported procedures[1] were followed. Briefly, small fragments of muscle tissue were trypsinized with $0.25 \%$ trypsin in calcium and magnesium free balanced salt solution $(\mathrm{CMF}), 37^{\circ} \mathrm{C}, 25 \mathrm{~min}$., then dispersed by repeated pipeting in culture medium $(10 \%$ horse serum, $10 \%$ embryo extract, $50 \mu / \mathrm{ml}$ penicillin, $50 \mu \mathrm{g} / \mathrm{ml}$ streptomycin and $250 \mu \mathrm{g} /$ $\mathrm{ml}$ fungizone in Eagle's minimum essential medium with Earles salts, Gibco). Cell suspension was passed through doubled thickness of lens paper in a Swinney adapter. Cells were plated onto plastic coverslips covered with collagen in $35 \mathrm{~mm}$ tissue culture dish at $4.5 \times 10^{5}$ cells / dish.

\section{Antibodies}

Anti-tubulin antibodies were obtained from polyclonal antiserum agrinst chicken embryo brain tubulin (Miles Co., USA). Specificity of the antiserum was tested by indirect immunofluorescence tests on cells. Anti-MHC an bibody was a monoclonal antibody (Mab) against chicken skeletal muscle myosin heavy chains (MHC). It was a gift from Dr. Frank Pepe (Department of Anatomy, School of Medicine, Universiby of Pennsylvania). Mab of MHC binds exclusively to A-bands of skeletal - and cardiac - myofibrils and does not bind to myosin in smooth muscle, presumptive myoblasts or other cell types examined [1]. Goat anti-rabbit or goat anti-mouse IgG antisera conjugabed either with FITC or Rhodamine fluorochrome were purchased from Cappel Co. (USA).

\section{Immunofluorescent staining procedures}

Anti-tubulin[6] : Coverslips were rinsed with warm EMS (2[N-Morpholino] ethanosuIronic acid, Sigma Co.) buffer, (MES, $0.1 M ; \mathrm{MgCl}_{2} 1 \mathrm{~m} M$; EGTA $1 \mathrm{mM}$; $\mathrm{PEG}_{8000} 4 \%$; $\mathrm{pH}$ 6.75), extracted in $0.2 \%$ Triton X-100 diluted with MES buffer for $2 \mathrm{~min}$; rinsed in MES buffer, then fixed with $2 \%$ glutaraldehyde diluted with MES buffer for $15 \mathrm{~min}$. at room temperature. After fixation, coverslips wore rinsed in PBS three times, then treated with $\mathrm{NaBH}_{4}$ (sodium borohydride, Fisher Sci. Co., $1 \mathrm{mg} / \mathrm{ml}$ in PBS) for $4 \mathrm{~min}$. three times; then rinsed in PBS before antibody incubation. Antibody incubation was carried out in humidified chamber, $37^{\circ} \mathrm{C}, 1 \mathrm{hr}$. Three washes in $0.5 \%$ Triton X-100 in PBS for a total of $30 \mathrm{~min}$. were necessary between antibody incubations. DNA-binding Bisbenzamide (Hoechst 33258 or $33342,10 \mu \mathrm{g} / \mathrm{ml}$ ) was used for nuclear staining.

Double staining for tubulin and MHC: Coverslips were rinsed with warm MES buffer, extracted in $0.2 \%$ Triton X-100 in MES buffer for 2 min., then fixed in $0.2 \%$ formaldehyde in MES buffer for 3min., then washed with PBS three times. Additional extraction in $0.5 \%$ Triton X-100 in PBS three times for a total of 30 min. was necessary before antisera incuabtion, also three washes with $0.5 \%$ Tribon X-100 in PBS for $30 \mathrm{~min}$. between each antiserum incubation.

\section{Electron microscopy}

For EM, cells were prepared as previously described [6].

\section{Chemicals}

TPA: a gift from Dr. T. G. O., Brien; taxol: kindly supplied from National Products 
Microtubules and my ofibrillogenesis

Branch, Division of Cancer Treatment, NIH, USA; coloemid: Sigmma Co.

\section{TPA treatment}

TPA-myosacs were obtained by treating day-3 muscle culture with TPA containing medium (final concentration $75 \mathrm{ng} / \mathrm{ml}$ ) for $48 \mathrm{hr}$.

\section{RESULTS}

\section{MTs and myofibrillogenesis in normal myoblasts}

There are mono-nucleated and multi-nucleated myoblasts in normal muscle cultures. In mono-nucleated myoblasts, the organization of MTs is identical to that in fibroblasts [6] with long, fine MTs radiating out from miorotubulo organizing center (MTOC) near the nucleus. MHC-fluorescence was found in postmitotic myoblasts. Multinucleated myotubes, formed through fusion of mono-nueleated postmitotic myoblasts, are thin and sparse in day-1 culture, and become increased in size and number later. Spontaneous contraction of single myotubes of day-3 culture and in most myotubes of day-4 cultures was observed. Myobubes of day-7 to day- 8 cultures are considered mature in respect to their size, well striated myofibrils, and contraction activities. During the developing process, mono-nucleated myoblasts tended to adhere to lateral edges of growing myotubes. MTs in these growing myotubes were numerous, fine and long, running parallel to the length of myotubes. MTOC were not seen. MHC-positive myofibrils were emerging in growing myotubes. Double staining with anti-MHG and anti-tubulin antibodies labeled with FITG and Rhodamine respectively showed a few striated myofibrils running together with many non-striated MHC-positive filaments over the length of immature myotubes. These nascent myofibrils co-localized with MTs and looked like lying on a background of MTs. MTs were numerous and extended beyond the lengbh of myofibrils in growth tips of developing myotubes ( Fig. 1a-d). Mature myotubes of day-8 muscle cultures were filled with well-aligned striated myofibrils. They co-localized with MTs which were rich and overlapping in cylindrical myotube that it was hard to identify a single MT.

\section{MTs and myofibrillogenesis in TPA-treated myoblasts}

Young developing myotubes of day-3 cultures exposed to TPA $(75 \mathrm{ng} / \mathrm{ml})$ for $48 \mathrm{hr}$. formed large, multi-nucleated, sac-shaped myocytes, TPA-myosacs. The number of nuclei in such myosacs varied from several to over 100. Those nuclei bend to cluster in central area of myosacs and commonly assume a ring-like arrangement. Those myosacs lack myofibrils but possess numerous MTs organized in radiating pattern. When TPA-myosacs were transforred to normal medium, myofibrillogenesis recovered in more or less synchronized manner in flattened myosacs where bho detailed distribution of both structures was easier to be seen. These myofibril-depleted myosacs began to synthesize their myofibrillar proteins and reassembled them into numerous long, well-aligned myofibrils. The influence of MTs on myofibrillogenesis was followed by allowing TPAmyosacs to recover in coloemid - or taxol containing medium in comparison with the recovery in normal medium in a time course from $3 \mathrm{hr}$. to as long as 6 days. 


\section{TPA-myosacs}

Elaborate assembly of MTs was observed in anti-tubulin stained TPA-myosacs. Numerous fine, long MTs radiated out from perinuclear area to cytoplasm, and a number of them approached to cell border. Intense tubulin fluorescence concentrated in the central area of cytoplasm (Fig. 2a) (Fig. 3a in ref. [7]). Striated myofibrils were dissociated into MHC-positive amorphous patches and were gradually depleted [7] (Micrograph not shown). In $48 \mathrm{hr}$. TPA-myosacs, none of the myofibrils except MHC-patches were left. Most of these MHC-patehes disappeared in $72 \mathrm{hr}$. myosacs [7].

\section{Recovery in normal medium}

By 3 hr., myosacs elongated slightly. MT was still in a radiating distribution but displaying a tendency to elongate (Fig. 2b), while MHC-positive myofibrils were not seen. During 16-24 hr. recovery, myosacs elongated further and formed cytoplasmic processes. Most of the cytoplasmic MTs were distributed parallel to longitudinal axis and were specially prominent in cytoplasmic elongating process (Fig. 2c). A few nascent MHCpositive filaments as a sign of recovering myofibrillogenesis were shown. Amorphous MHCpatched remnants sometimes co-existed in early recovering myosacs (Fig. 2d). By 48 hr: recovery, myosacs became further elongated, resembling normal day-3 myotubes, but were more flattened. MTs were long, fine and numerous. They ran parallel to myotubular longitudinal axis, and extended to the very ends of growth tips far beyond the extension of growing myofibrils. Newly formed MHC-myofibrils, some non-striated and filamentous while some striated, were increased in number and length. They co-localized with preexisting MTs and ended at the base of growth tips (Fig. 2e, 2f). This phenomenum, as well as that in normal developing myotubes, suggested that MTs might play roles as scaffold to support and guide the bipolar elongation of developing myofibrils. By 3 day recovery, myotubes were comparable to those in day-7 to day-8 normal cultures. Co-localization of myofibrils and MTs was conspicuous in the belly of myobubes. However, MTs extended farther than myofibrils at growth tips.

\section{Recovery in taxol-containing medium}

Recovery of TPA-myosacs in taxol (MT stabilizing reagent, final concentration $10 \mu M$ ) containing medium was followed at the time course from $3 \mathrm{hr}$. to 6 days. Double staining of tubulin and MHC immunofluorescence showed that numerous short MT-bundles formed in TPA-myosacs exposed to taxol medium for $3 \mathrm{hr}$., while MHC-myofibrils did not show up. Taxol induced MT bundles surrounded nuclear clusters to form irregular "rosette" figures (Fig. 3a) in $3 \mathrm{hr}$. taxol recovering myosacs and MT streaks became longer and distributed discretely in the cytoplasm of $24 \mathrm{hr}$ taxol recovering myosacs (Fig. 30). Noteworthy was the programmed myofibrillogenesis in recovering myosacs in taxol medium did proceed on schedule as well as those recovered in normal medium. Interesting]y, newly formed short MHC-myofibrils co-localized precisely with these MT-streaks (Fig. 3d, arrows). For further recovery in taxol medium, myosacs did not elongate properly. Though myofibrils became striated, increased in number, and contracted Occasionally, they did not elongate properly. EM investigation revealed that normal myofibrillar crystal-hexagonal 


\section{Microtubules and my ofibrillogenesis}

transverse structures were interspersed by abundant MTs which intermingled or interdigitated with MHC thick filaments (Fig. 3b). After 6 day exposure in taxol medium, myofibrils looked like mature normal myofibrils with clearcut striations (Fig. 3e, 3f). They co-localized with MT streaks, both were bipolarly orientated in myosacs. EM observations showed that in some area of the myosacs, the I-Z-I complexes of myofibrils were replaced by MTs [1]. In summary, TPA-myosacs never elongated and recovered to normal myotube shape even they stayed in taxol medium as long as 6 days.

\section{Recovery in colcemid containing medium}

TPA-myosacs after released from TPA were shifted immediately into colcemid ( $1 \mu \mathrm{M})$ containing medium. Myofibrillogenesis in the absence of polymerized MTs was followed at the time course from $2 \mathrm{hr}$. to 6 days.

As shown in Fig. 4, the recovering myosacs in colcemid medium became even thicker comparing with untreated TPA-myosacs. Double staining with anti-tubulin and antiMHC antibodies revealed that instead of polymerized MTs, diffused tubulin fluorescence was distributed in the cytoplasm. Tangled, non-striated MHC-positive filaments emerged in $24 \mathrm{hr}$. colcemid treated myosacs. By $48 \mathrm{hr}$. in colcemid medium, most myosacs were filled with striated MHC-myofibrils (Fig. 4a), some of them were aligned and some were in an intermingled state. However, neither did myosacs nor their developing myofibrils elongate properly in colcemid medium. The striations of myofibrils became more evident with prolonged stay in colcemid (Fig. 4b). Spontaneous twitching was observed occasionally in long-term (2-4 days) colcemid treated myosacs. However, TPA-myosacs never elongated and recovered to normal myotube in coleemid medium.

\section{DISCUSSION}

This work confirmed the earlier demonstrations [4] that intact MTs are required for normal elongation of myotubes and the deployment of thick filaments during myofibril formation. The observations in this report, which include (1) the precedence of bipolar orientation of MTs in early fused myotubes and in recovering TPA-myosacs, and (2) the co-localization of emerging myofibrils and MTs indicate further the importance of MT orientation in conducting myofibril alignment during myofibrillogenesis.

The centers or organelles responsible for the arrangement of MT distribution in myogenic cells would be very important in contributing to the bipolarity of developing myotubes. It has been repeatedly demonstrated that the switch on of differentiation programs from replicating presumptive myoblasts to their daughter postmitotio definitive myoblasts occurs prior to fusion. Change of MT distribution pattern from essentially radiating in mono-nucleated myoblasts to parallel in fused multi-nucleated myotubes suggests that mechanisms controlling MT organization are subject to conformational differentiation process in myogenic cells. The MT organizing centers (MTOCS) were dispersed and disappeared gradually during maturation of myogenic cells. It has been reported that the MT nucleating materials have been redistributed from pericentriolar area to nuclear periphery during myofibrillogenesis[8]. The taxol induced tremendous number bf short MT bundles at nuclear periphery area in early recovering TPA-myosacs was also 
ebserved (Fig. 3a).

The remarkable co-localization between MTs and myofibrils implies the possibility of interactions between them. Electron dense Liking bridges were noted between MT-MT and MT-thick filaments here and there (Fig. $3 \mathrm{~b}$ ) which confirmed previous observations [4, 6]. Hayashi et al. [9] have reported that myosin and tubulin co-precipitate at low ionic strength; myosin filaments with cross bridges which were decorated by tubulin aggregates side by side; 1 mol of myosin binds 2 reels of tubulin dimer. However, accurate structural evidence of linking bridges in vivo is needed.

Both taxol and colcemid not only block the normal elongation of myofibrils and myotubes, but also interfere with the assembly of forming myofibrils [4, 10]. Taxolinduced stabilization and increase of MTs number has mainly changed the normal ratio among MTs, thick filaments and thin filaments in the limited space of sarcoplasm $[4,10]$. In $48 \mathrm{hr}$. taxol treated TPA-myosacs, one thick filaments surrounded by 5-6 single MTs was very common (Fig. 3b). This increased ratio of MTs to both thick-and thin filaments is one of the possibilities that account for the formation of arrays of interdigitating MTs and thick filaments [4]. Although colcemid-induced depolymerization of MT and changes of myofibrils were different from those of taxol, both drugs broke the balanced steady dynamic state of MTs[11] which might be essential for normal myofibril formation in myogenic cells.

TPA-myosacs recovered in either normal, taxol or colcemid medium, most significantly during 24-48 hr. recovery period. Developing myofibrils emerged around $24 \mathrm{hr}$. and matured after $48 \mathrm{hr}$. recovery [2] (Fig. 2, 3 and 4). Neither of the drug-induced changes including stabilization and depolymerization of MTs altered the scheduled program of myofibril formation. It is most likely that the influence of MTs in myofibrillogenesis is post-translational. In other words, MTs might be involved in spatial deployment of the myofibrillar proteins in myofibrillogenesis[4].

\section{ACKNO WLEDGEMENT}

We are indebted to Dr. John Murray (Department of Anatomy, School of Medicine, University of Pennsylvania, USA) for his help in EM work.

\section{REFERENCES}

[1] Antin PB, Tokunaka S, Nachmias VT, Holtzer H. Role of stress fiber-like structures in assembling nascent myofibrils in myosheets recovering from exposure to ethyl methanesulfonate. J Cell Biol 1986; 102:1464-79.

[2] Antin PB, Forry-Schaudies S, Tokunaka S, Duran A, Eshelman J, Holtzer H. Use of a cocarcinogen (TPA) and a carcinogen (EMS) to probe of myofibrillogenesis. In: Alan R. Liss, Inc. Molecular Biology of Muscle Development. 1986: 709-23.

[ 3 ] Holtzei H, Forry-Schaudies S, Dlugosz A, Antin P, Dubyak G. Interactions between IFs, microtubules and myofibrils in fibrogenic and myogenic cells. In: Intermediate Filaments. Ann NY Acad Sci 1985; 455:106-125.

[4] Antin P, Forry-Schaudies S, Friedman T, Tapscott S, Holtzer H. Taxol induces postmitotic myoblasts to assemble interdigitating microtubule-myosin arrays that exclude actin filaments. J Cell Biol 1981; 90:300-308.

[5] Saitoh O, Arai T, Obinata T. Distribution of microtubules and other cytoskeletal filaments during myotube elongation as revealed by fluorescence microscopy. Cell tissue Res 1988; 252:263-73.

[6] Forry-Schaudies S, Murray JM, Toyama Y, Holtzer H. Effects of colcemid and taxol on microtubules and intermediate filaments in chick embryo fibroblasts. Cell Motility and the cytoskeleton 1986; 6:324-38.

[ 7 ] Lin ZX, Eshelman JR, Forry-Schaudies S, Duran S, Lessard JL, Holtzer H. Sequential disassembly of myofibrils 


\section{Microtubules and my ofibrillogenesis}

induced by myristate acetate in cultured myotubes. J Cell Biol 1987; 105:1365-76.

[ 8 ] Tessin A-M Maro B, Bornens M. Fate of microtubule-organizing centers during myogenesis in vitro. J Cell Biol $1985 ; 100: 35-46$.

[ 9 ] Hayashi M, Ohnishi K, Hayashi K. Dense precipitate of brain tubulin with skeletal muscle myosin. J Biochem (Tokyo) 1980; 87:1347-55.

[10] Toyama Y, Forry-Schaudies S, Hoffman B, Holtzer H. Effects of taxol and eolcemid on myofibzillogenesis. Proc Natl Acad Sci USA 1982; 79:6556-60.

[11] Schulze F, Kirschner M. Microtubule dynamics in interphase cells. J Cell Biol 1986; 102:1020-B1.

\section{Recieved 16-1-1989. Revised16-3-1989. Accepted 12-6-1989.}

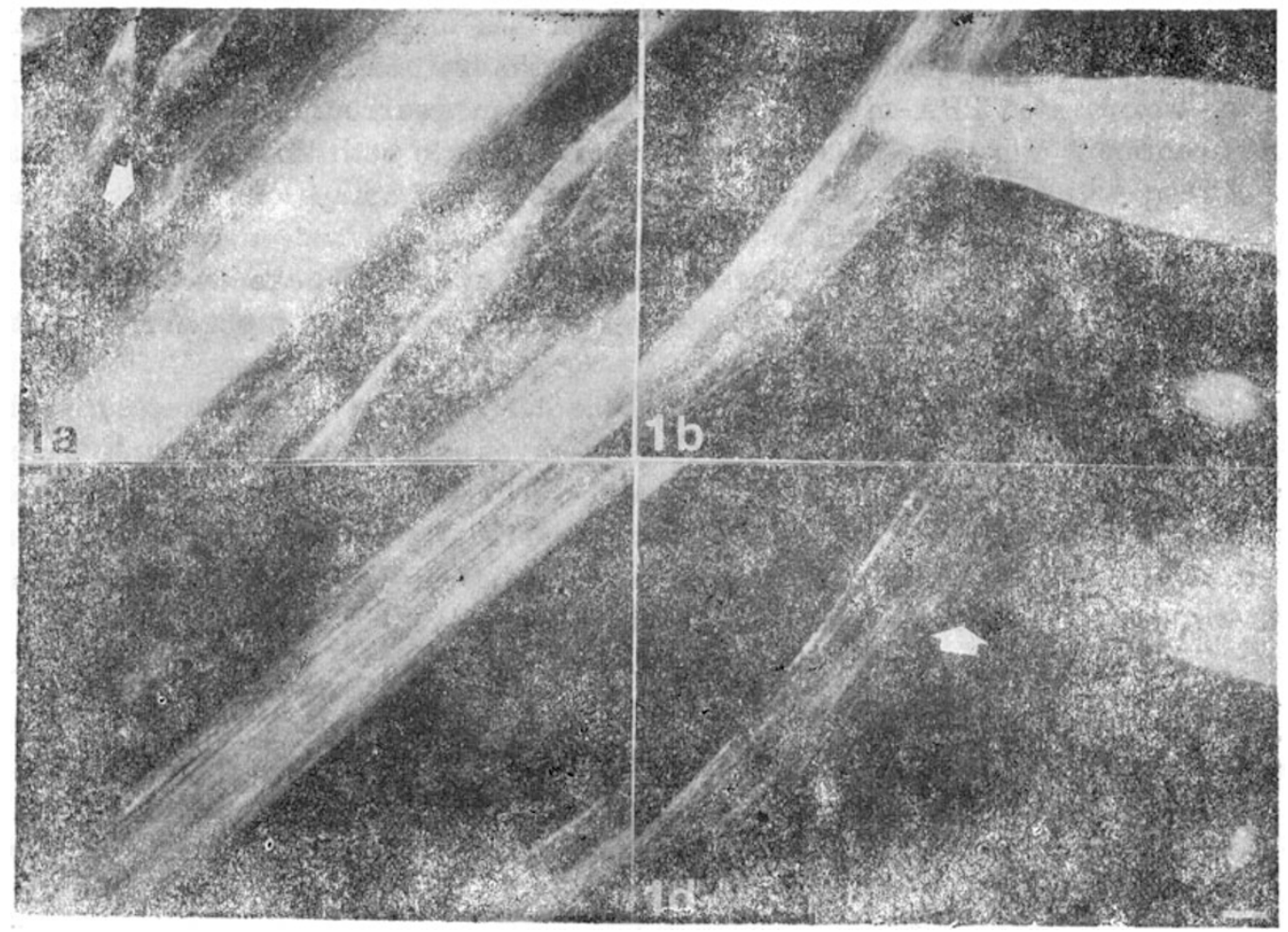

Fig 1. Immunofluorescent micrographs of myotubes from day-3 muscle culture showing double staining of anti-tubulin (a, b) and anti-MHC (c, d). Arrow in (a) pointing to an adhering myoblast. Compare (a) with (c), (b) with (d), striated myofibrils are emerging in these developing myotubes where NITs are numerous and are orientated in precedence. Arrow in "(d) showing the absence of MHC-myofibril in the growth tip of a myotube where MTs are abundaut (b). bar: $10 \mu \mathrm{m}$. 


\section{Lin ZX and H Holtzer}

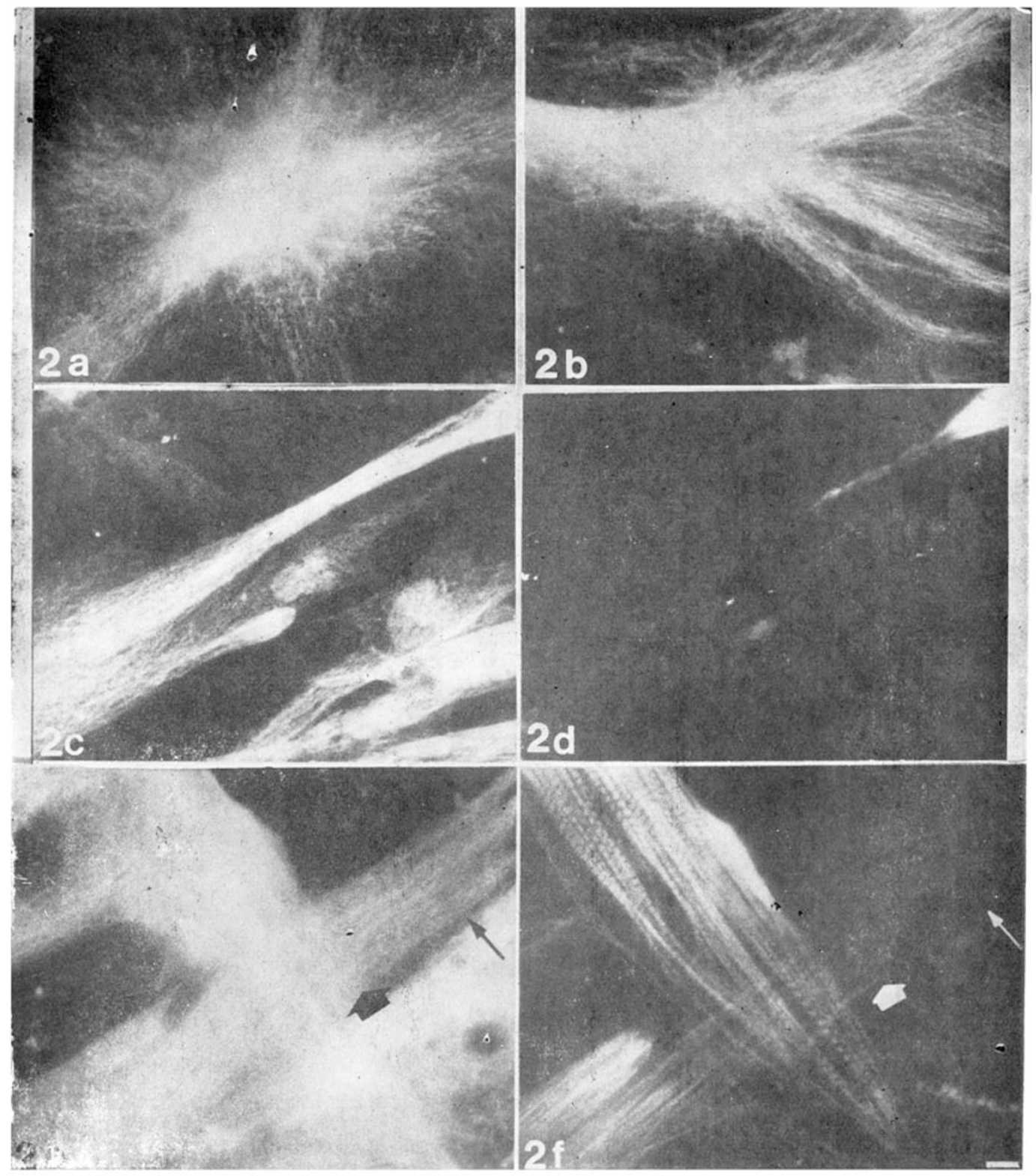

Fig 2. (a) Anti-tubulin immunofluorescent staining of a TPA-mypsac. (b)TPA-myosac recovered in normal medium for $3 \mathrm{hr}$. Anti-tubulin staining. (c)TPA-myosac recovered in normal medium for $16 \mathrm{hr}$. Anti-tubulin staining. (d) Same cell of (c), MHC immunofluorescent staining. The MHC-positive fluorescence at the end of a cytoprocess showed the degraded myofibrillar MHC patches. (e)TPA-myosac recovered in normal medium for 48 hr. Anti-tubulin stained MTs and (f) the same cell in (e), anti-MHC stained myofibrils. Remarkable co-localization between MTs in (e) and myofibrils in (f) is shown. Thin arrows in (e) and (f) pointing to a growth tip where only a few faintly stained MHC filameats ended at the base (broad arrow) while numerous MTs approached to the end (thin arrow). bar $10 \mu \mathrm{m}$. 


\section{Microtubules and my ofibrillogenesis}

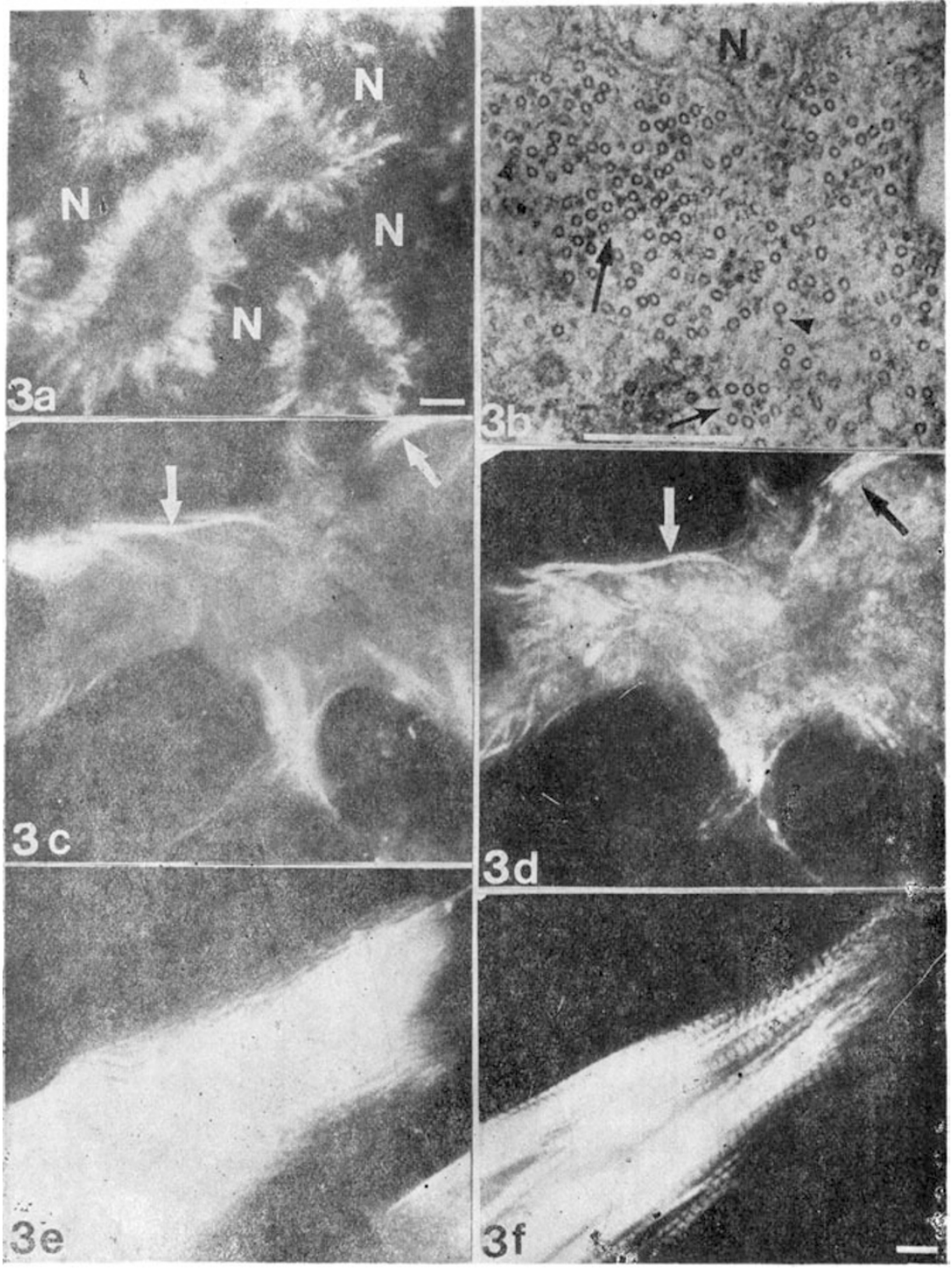

Fig 3. (a) TPA-myosac, recovered in taxol medium for $3 \mathrm{hr}$.; anti-tubulin immunofluorescence. (b) E1ectron micrograph of myosac recovered in taxol medium for $48 \mathrm{hr}$. showing interdigitating arrays of MTs and thick filaments (arrow) near the nucleus. Linkages between MT-MT and MT-thick filaments are shown here an4 there (arrow heads). (c, d) Same TPA-myosac recovered in taxol medium for $24 \mathrm{hr}$. (c)Anti-tubulin staining and (d) anti-MHC staining showing remarkable co-localization of early emerging single striated myofibrils with MT streaks (arrows). The bipolarity of the arrangement of MTs and MHC filaments are shown in most areas. (e, f) Same TPA-myosac recovered in taxol medium 6 days MTs axe abundant (e). C1earcut striated MHC myofibrils in (f) co-localized with MTs in (e). Eoth are bipolarly arranged but no sign of further e1ongation. bar: (a, c-f) : $10 \mu \mathrm{M}$. bar(b): $0.5 \mu \mathrm{m}$. 


\section{Lin ZX and H Holtzer}

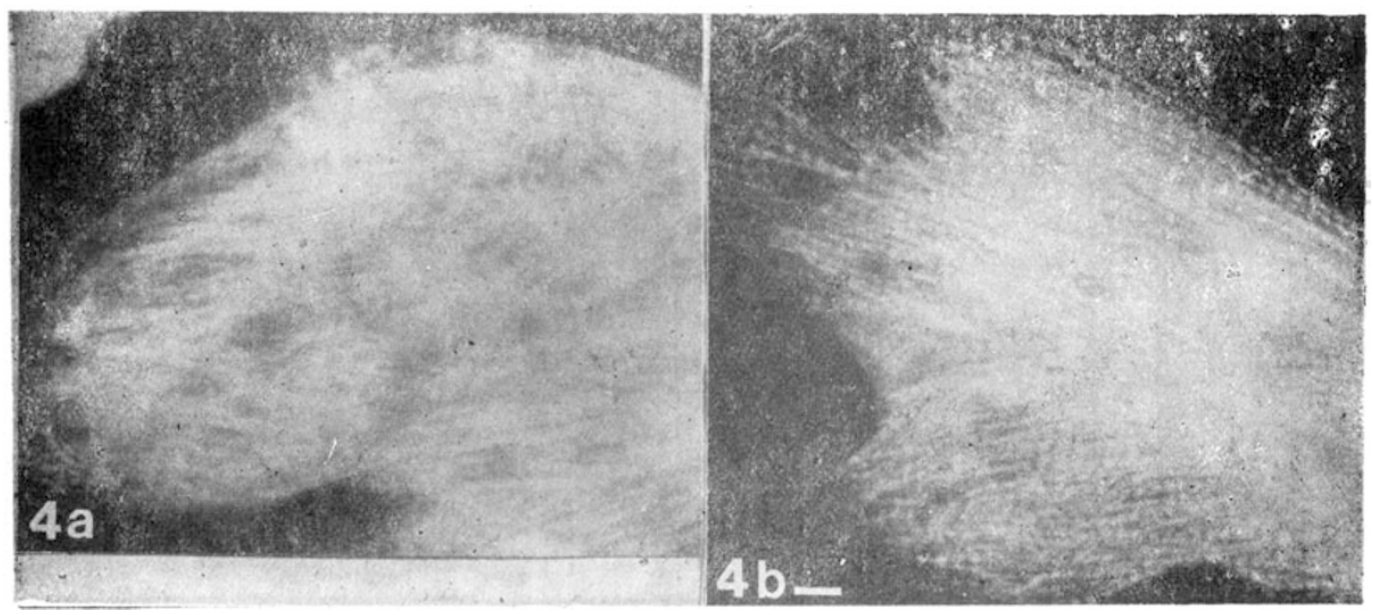

Fig 4. MHC immunofluorescence in TPA-myosac recovered in colcemid medium for 48 hr. (a), and S days (b). Tangled non-striated and striated MHC filaments are shown in $48 \mathrm{hr}$ colcemid recovery (a); striated myofibrils, irregularly or intermingled aligned, are abundant in 3-day colcemid recovery (b). The myosac did not elongate properly, neither did the included myofibrils, bar: $10 \mu \mathrm{m}$. 